\title{
Knowledge, attitude, and intentions towards fertility preservation in cancer patients among healthcare workers in Northern India
}

\author{
Neena Malhotra ${ }^{1}$, Monica Gupta ${ }^{1}$, Anshu Yadav ${ }^{1}$, Perumail Vanamail ${ }^{1}$, Reeta Mahey ${ }^{1}$ \\ ${ }^{1}$ Department of Obstetrics \& Gynecology, All India Institute of Medical Sciences, New Delhi, India
}

\begin{abstract}
Objective: To study the knowledge, attitude, and intentions towards fertility preservation (FP) in cancer patients among healthcare workers (HCWs) in Northern India.

Methods: The survey discussed in this study was a 23-item structured questionnaire on oncofertility designed based on previous studies on the topic. A link to the questionnaire was sent through WhatsApp to healthcare workers involved in the care of cancer patients. Their responses were analyzed subsequently.

Results: More than a third (37.7\%) of the $750 \mathrm{HCWs}$ contacted answered the questionnaire. The group included gynecologists, surgeons, and oncologists. Although $90 \%$ of the respondents were aware of the harmful effects of cancer therapy on ovarian function, $76 \%$ claimed they had only partial knowledge about fertility preservation (FP). Only a fourth of the respondents were aware of the time needed for oocyte cryopreservation and a third had knowledge of the number of semen samples required for FP. Among HCWs involved in the care of young cancer patients, only $50 \%$ reported that they had referred patients for FP. The most common reason for not referring patients for FP was lack of knowledge about FP (43\%). More than $90 \%$ claimed they wanted to improve their knowledge about oncofertility through continued medical education programs and seminars.

Conclusions: The study emphasizes the need for establishing well-structured networks to improve knowledge about FP among HCWs, so that cancer patients are offered the chance of using their gametes to have children after they have been cured from cancer.
\end{abstract}

Keywords: cancer, fertility preservation, gonadotoxic, oncofertility, healthcare workers

\section{INTRODUCTION}

The incidence of cancer is increasing worldwide. According to data published by the World Health Organization (WHO) in 2018, 9.6 million or one in six deaths were due to cancer (WHO, 2020). GLOBOCAN 2018 reported breast cancer as the most common cancer in the general population (14\%) and among females $(27.4 \%)$, followed by cervical and ovarian cancer (Bray et al., 2018). The incidence of breast cancer among younger Indian women is increasing when compared with Western countries (Parmar, 2018). Prevalence of breast cancer is predicted to increase to 35 per 100,000 women in India in 2035 (Mathur et al., 2020). With the rising incidence of breast and other cancer types, fertility concerns among young cancer survivors are expected to increase proportionately in the Indian subcontinent.

According to the 2018 Guidelines of American Society of Clinical Oncology (ASCO) and the American Society of Reproductive Medicine (ASRM), healthcare workers (HCWs) involved in the care of adult and pediatric cancer patients should address the possibility of infertility as early as possible before the start of treatment and include the possible options of fertility preservation (FP) in the discussion (Oktay et al., 2018). Since time is a crucial element in the management of these patients, fertility counseling and referral to FP should be considered as soon as patients are diagnosed with cancer instead of when they are started on gonadotoxic therapies.

There is an overall lack of awareness among clinicians about the detrimental effects of cancer treatment on fertility, FP options, the timing to refer patients to FP, the times involved in FP procedures, and post procedure implications. Most of the studies performed in Western countries have described poor knowledge, attitude, and awareness of oncofertility among clinicians (Sallem et al., 2018; Chehin et al., 2017). Although FP options abound, oncofertility resources have been underutilized and the target population has largely missed out on the chance to have their gametes frozen for purposes of having future parenthood experiences (Woodard et al., 2018).

Ours is a tertiary care referral hospital in Northern India that caters to the needs of cancer patients from all over the country. As the number of young cancer survivors in India is on the rise and there is sparse data about oncofertility practices and networks in India, the present cross-sectional study was designed to evaluate the knowledge, attitude, and intentions of HCWs involved in the care of cancer patients regarding the effects of cancer treatment on fertility and fertility preservation options available. The proposed methods to improve the knowledge and awareness of HCWs in this field are also discussed.

\section{MATERIAL AND METHODS \\ Study design}

The present cross-sectional study was conducted at a tertiary care center (September 2019 to March 2020) after approval from the Institute Review Board (IEC566/02.08.2019).

\section{Questionnaire design}

To design the questionnaire, a literature search was done on Pubmed and Google Scholar and articles were searched based on keywords "fertility preservation", "oncofertility", and "knowledge and awareness among clinicians". The next step entailed the organization of a Focus Group Discussion (FGD), during which questionnaire items were developed. Previous studies were reviewed, and questions were designed according to our setup. The questionnaire was tested initially with $10 \mathrm{HCWs}$ ( 7 gynecologists and 3 oncologists) to check the validity and ease of understanding the questions. Attention was given to keeping the questions in proper sequence and using simple language to allow ease of understanding. After approval, it was sent via mobile messaging application (WhatsApp) to clinicians from the Gynecology, Surgery, and Oncology departments. A brief explanation and the reasons for the survey were delivered in an initial message, which was followed by another message with the link to the 
questionnaire. Participants answered the questionnaire voluntarily. The questionnaire comprised 23 items and included information about healthcare worker demographics, clinical practice experience, knowledge and awareness of oncofertility as a sub-specialty, and intentions and attitude towards acquiring more knowledge about the field. Participants were asked to provide their email addresses and the rest of the information was anonymized.

\section{Data collection}

The questionnaire was sent to HCWs (gynecologists, surgeons, and oncologists - medical, surgical, and radiation oncologists) involved in the care of cancer patients in Northern India. All the information from the forms completed by the participants was combined in a central database and pre-specified variables were analyzed.

\section{Statistical analysis}

A database was developed using Microsoft Excel and analysis was performed using SPSS IBM version 22.0 (Armonk, NY, IBM Corp.). For normally distributed data, descriptive measures such as mean and standard deviation were calculated. Categorical variables were expressed as frequency and percent values and compared using the Chisquared/Fisher's exact test. Post-hoc analysis was carried out for multiple comparisons using the Bonferroni correction. For all statistical tests, a two-sided probability of $p<0.05$ was considered for statistical significance.

\section{RESULTS}

A total of $750 \mathrm{HCW}$ from different specialties involved in the care of cancer patients were sent text messages through WhatsApp with the link to the questionnaire; 287 answered the questionnaire, yielding a response rate of $37.7 \%$.

\section{Demographic characteristics}

Respondent characteristics are shown in Table 1. Ninety-seven of the $283(34.2 \%)$ were gynecologists; $78(27.5 \%)$ were medical oncologists; 58 were surgeons $(20.5 \%) ; 31$ were surgical oncologists $(10.9 \%)$; and 19 were radiation oncologists $(6.7 \%)$. About $61.1 \%$ of the participants were aged $26-35$ years $(n=173)$. The female to male ratio was $1.6: 1$ ( $62.8 \%$ vs. $37.1 \%)$. Almost half of the respondents had been in clinical practice for less than 5 years $(47 \%)$ and only $70(24.7 \%)$ had been more than 10 years of clinical experience. The most common cancers dealt with by the respondents were gynecological tumors $(78.2 \%)$, followed by breast, gastrointestinal, urological, hematologic, musculoskeletal, pediatric, and testicular cancer. Almost $83 \%$ (236) claimed that they dealt with young cancer patients needing gonadotoxic therapy.

\section{Knowledge and awareness}

More than $90 \% \quad(n=271)$ of the respondents were aware that cancer treatment affects future reproduction capabilities. Almost $90 \%(n=252)$ were well informed about the association between ovarian function reduction and chemotherapy. Almost $90 \%(n=253)$ were aware that among the reproductive organs, the ovaries are more significantly affected by chemotherapy. However, only $44 \%$ of the respondents correctly identified cyclophosphamide as the most gonadotoxic chemotherapy agent.

When enquired about their knowledge of FP, almost $77 \%$ $(n=217)$ claimed they had partial knowledge about FP in cancer patients (Figure 1$)$. Eighty-six percent $(n=245)$ of the respondents were aware that patients need FP procedures prior to the start of gonadotoxic chemotherapy. But when enquired about the upper age limit for FP in females, only $40 \%$ correctly answered that 40 years was the upper age limit.

\begin{tabular}{|c|c|}
\hline \multicolumn{2}{|c|}{ Table 1. Characteristics of healthcare workers. } \\
\hline Characteristics & N (\%) \\
\hline Healthcare workers contacted & 750 \\
\hline Total responses & $283(37.7 \%)$ \\
\hline Gender & $105(37.1 \%)$ \\
Male & $178(62.8)$ \\
Female & $16(5.7 \%)$ \\
\hline Age (years) & $173(61.1 \%)$ \\
$20-25$ & $69(24.4 \%)$ \\
$26-35$ & $25(8.8 \%)$ \\
$36-45$ & $97(34.2 \%)$ \\
$>45$ & $78(27.5 \%)$ \\
Gynecology & $58(20.5 \%)$ \\
Medical Oncology & $31(10.9 \%)$ \\
Surgery & $19(6.7 \%)$ \\
Surgical Oncology & $133(47 \%)$ \\
Radiation Oncology & $80(28.3 \%)$ \\
\hline Specialty & $70(24.7 \%)$ \\
\hline Experience (years) & \\
$6-10$ &
\end{tabular}

Only $25 \%(n=71)$ correctly answered that $10-12$ days are needed for oocyte or embryo cryopreservation (Figure $1)$. Almost two thirds $(67 \%)$ of the respondents did not know the number of semen samples needed for male fertility preservation (Figure 1 ). Regarding FP methods, oocyte $(86.2 \%)$ and semen cryopreservation (71.6\%) were among the best known methods (Figure 2).

\section{Attitude and intentions}

Just over half $(53 \%)$ of the respondents $(n=150)$ routinely referred young cancer patients for FP counseling. The remaining respondents $(47 \%)$ claimed that lack of knowledge was the main reason why they did not refer patients to FP $(43 \% ; n=58)$. Other common reasons included the inability of patients to afford FP procedures $(22.5 \%$, $\mathrm{n}=30$ ) and the lack of knowledge about referral pathways (16.5\%, $n=22$ ). Lack of time to counsel patients, marital status (unmarried), and fear of transmitting cancer to their offspring were the least common reasons for not referring patients. More than $90 \%$ of the participants $(n=260)$ were desirous of improving their knowledge about FP. The most common paths to improving knowledge about the subject were continued medical education $(33.8 \%)$, seminars $(31.3 \%)$, and informative leaflets $(23.9 \%)$. Almost $97.5 \%$ $(n=276)$ of the respondents were willing to join a second follow-up survey. No difference in knowledge was found among clinicians based on years of clinical experience.

\section{DISCUSSION}

The present study found low levels of knowledge and awareness about oncofertility among HCWs in Northern India.

There are global barriers in the field of oncofertility that need to be addressed and overcome by collaboration with professional societies and governments. Another paper published previously by our group found poor overall levels of knowledge among cancer patients about the gonadotoxic effect of cancer therapy and available FP options (Mahey et al., 2020).

Although response rates are usually low, most of the studies about knowledge and awareness among clinicians are based on surveys sent by email or social media links 

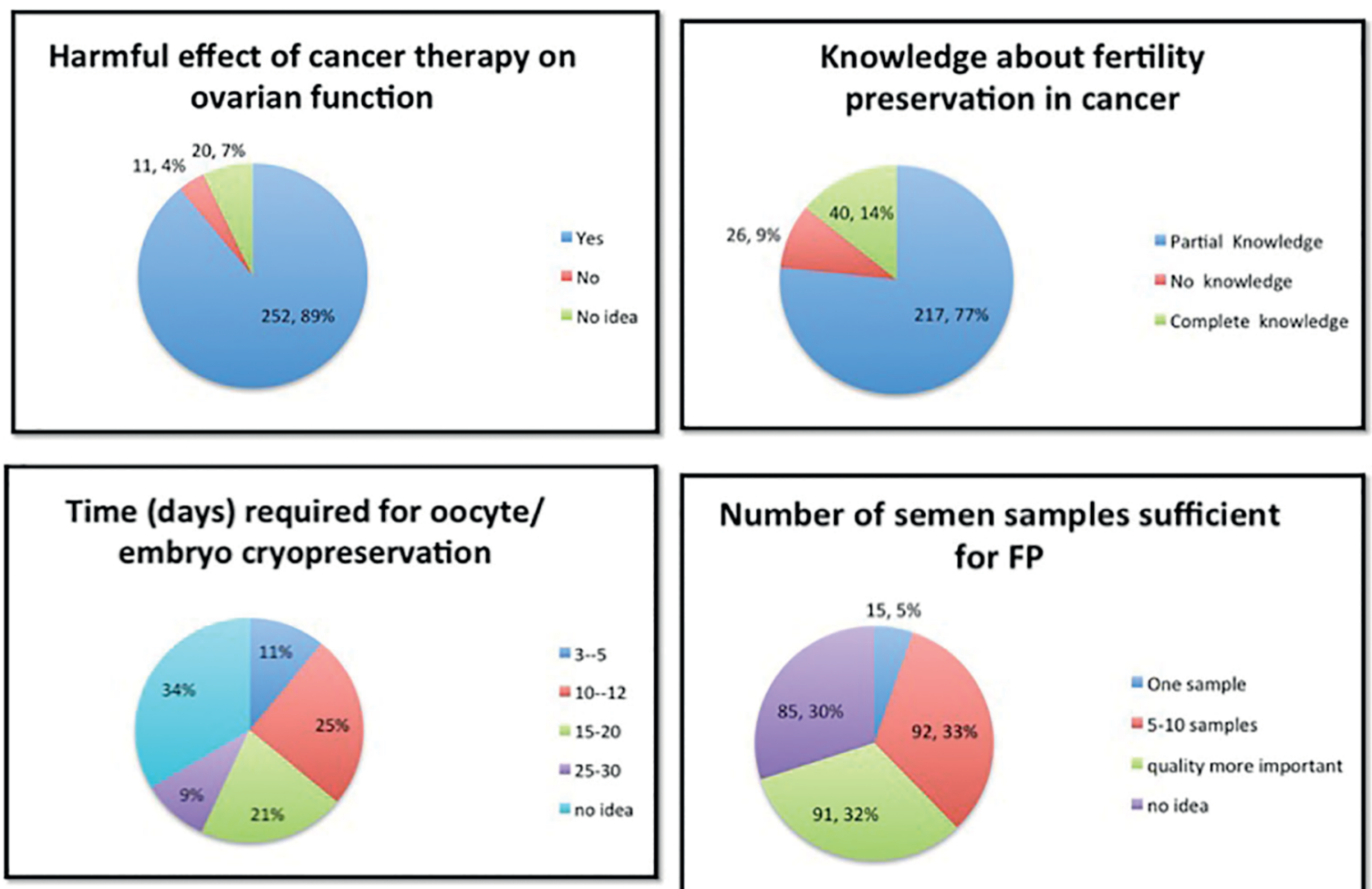

Number of semen samples sufficient for FP

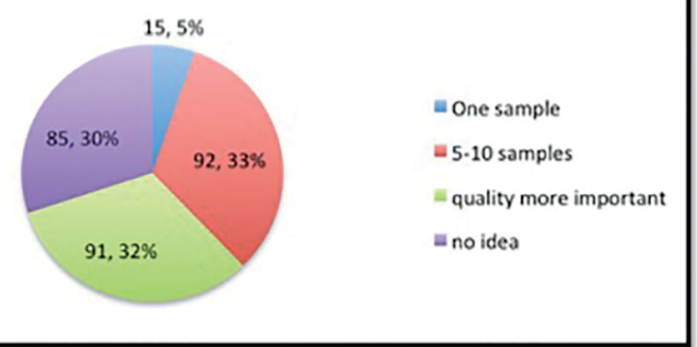

Figure 1. Percentage response regarding knowledge about different options of fertility preservation (FP).

\section{Fertility options}

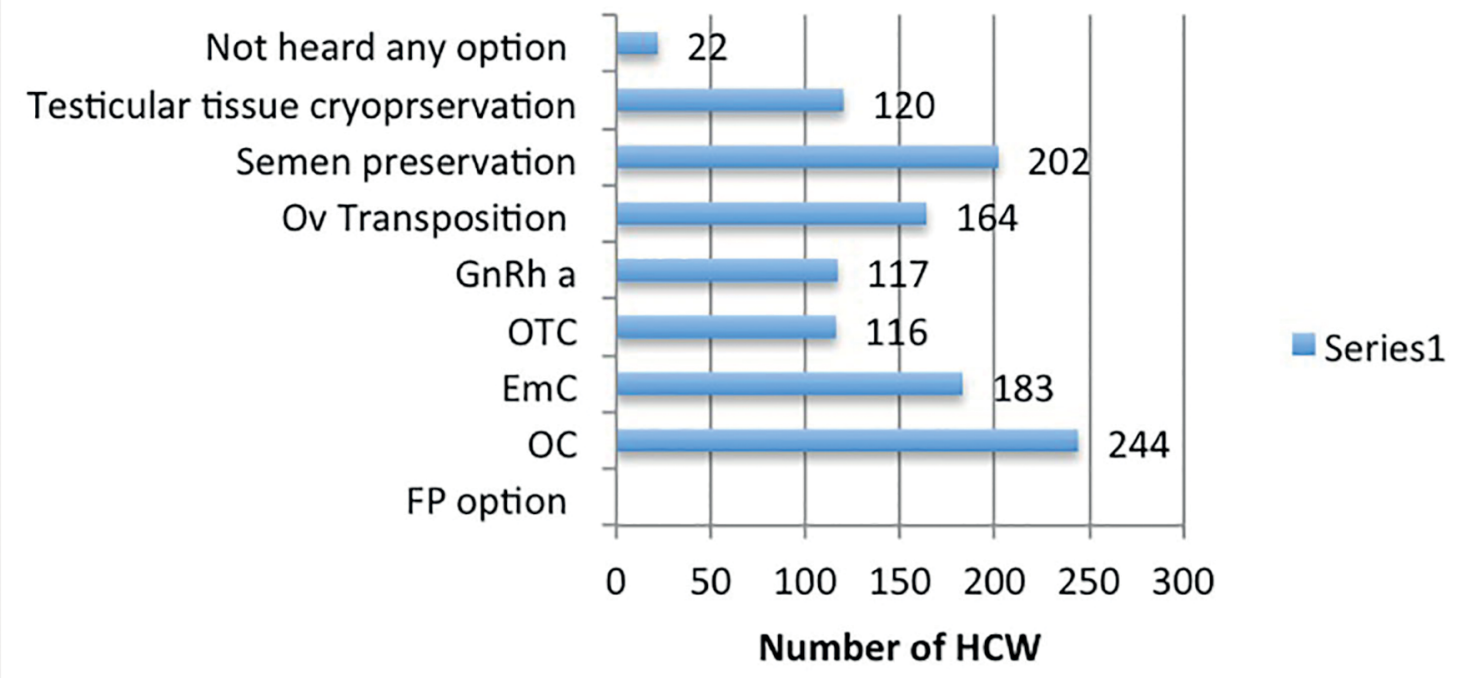

Figure 2. Knowledge regarding various fertility preservation strategies. Abbreviation: Ov transposition, ovarian transposition; OTC, ovarian tissue cryopreservation; EmC, embryo cryopreservation; OC, oocyte cryopreservation; GnRHa, gonadotropin releasing hormone agonist, HCW, health care worker; FP, fertility preservation. 
(Sallem et al., 2018; Chehin et al., 2017). A recent global survey with physicians about FP options available to patients had a response rate of only $25 \%$ (Rashedi et al., 2020). The present study had a higher, although not entirely satisfactory, response rate. The study was stopped at end of six months. As in other studies developed in India (Mahajan et al., 2016), the number of female responders was significantly higher than the number of male respondents, possibly because women are more concerned with fertility and parenthood.

Oncologists generally resist discussing FP options, with reasons ranging from the need to treat cancer patients without any delay, a lack of awareness about recent developments in oncofertility, to lack of time (Knight et al., 2015). Lack of knowledge was the most commonly reported reason for not referring patients to FP. The findings of this survey may also be extrapolated to the general population of physicians in India, whose knowledge of the subject is lesser than that of physicians working in tertiary care referral hospitals due to limited access to recent updates in this field of medicine. Table 2 shows the gaps in oncofertility referrals and key steps to improve the knowledge of HCWs in our country.

Since lack of time has been reported as of the major reasons for not referring patients to FP, other HCWs such as nurses and counselors who also come in contact with young cancer patients during treatment may play an important role at advising patients about the effects of cancer therapy, educating them about FP, scheduling consultations with fertility clinicians, and discussing financial implications.
Our survey found that HCWs had little knowledge about the time needed for oocyte/embryo freezing and the number of semen samples required for FP. There is a need to develop structured networks to improve knowledge about oncofertility in each level, so that timely referral is made possible. We need to explore the social, ethical, religious, and financial factors affecting FP decisions among patients. Financials factors are typically important in low-to-middleincome countries (LMIC) like ours, since FP procedures are not offered free of charge or covered by insurance, which means that families must bear the expense (Rashedi et al., 2020). Even in cases of timely referral to FP, some patients cannot undergo the procedure due to financial constraints and the short window they are allowed to make the required monetary arrangements.

The limitations of the study include the small size of the sample and the low response rate of participants contacted. We were unable to get responses from physicians of other specialties such as hematology. The study found overall meager knowledge about FP among HCWs and emphasized the need to spread awareness through different social media platforms and provide for an easy and approachable network to manage these patients. Support of professional societies and national platforms are desirable to establish oncofertility care so that the dream of parenthood may come true for cancer survivors.

\section{ACKNOWLEDGEMENTS}

We acknowledge and thank all the health care workers who took their valuable time to fill the questionnaire.

Table 2. Gaps in oncofertility services and proposed steps to improve knowledge and awareness in India.

\section{Gaps and concerns in the oncofertility services}

Poor knowledge among oncologists regarding fertility preservation (FP)

Lack of time

Patient concerns

Building awareness at a national level

Bridging the gap between cancer and fertility

\section{Key steps to improve the services}

1. Establish oncofertility clinics involving oncologists and reproductive medicine (RM) clinicians

o To plan FP procedures for patients in need.

o To discuss the type of cancer, survival rate, risk of gonadotoxicity from cancer therapy, desire for fertility preservation, time available, physical and medical fitness of patient to undergo FP procedure.

2. Organize FP seminars and continued medical education (CME) and produce leaflets so that information can be disseminated to cancer clinicians at a national level.

Involvement of RM clinicians/fellows to counsel patients at the time of initial diagnosis so that reproductive concerns may be taken care of.

o Other staff such as social workers and nurses can be trained to discuss fertility issues with patients. They may counsel patients in preliminary interviews and refer patients in need.

The procedure of gamete/embryo cryopreservation may be available at minimum or free of cost to patients, especially for those unable to bear the cost of the procedures.

Detailed patient counseling by the oncologist and RM clinician at the time of cancer diagnosis to allow timely referral, so that patient and family concerns are addressed and FP is performed without delaying cancer treatment.

Use social media platforms to provide an easy and approachable network to manage these patients. Support of professional societies and national bodies can be sought to organize conferences at a national level for wider dissemination of information.

\section{Establish an Oncofertility Consortium}

o Multidisciplinary team comprising medical specialists (e.g., oncologists, hematologists, reproductive endocrinologists, urologists, surgeons, pathologists) and healthcare staff including nurses and genetic, mental health and social counselors, and the embryology/andrology lab team stakeholders 


\section{CONFLICT OF INTERESTS}

The authors have no conflict of interest to declare.

\section{Corresponding author:}

Reeta Mahey

Department of Obstetrics \& Gynecology

All India Institute of Medical Sciences

New Delhi - India

E-mail: reetamahey52@gmail.com

\section{REFERENCES}

Bray F, Ferlay J, Soerjomataram I, Siegel RL, Torre LA, Jemal A. Global cancer statistics 2018: GLOBOCAN estimates of incidence and mortality worldwide for 36 cancers in 185 countries. CA Cancer J Clin. 2018;68:394-424. PMID: 30207593 DOI: 10.3322/caac. 21492

Chehin MB, Bonetti TC, Serafini PC, Motta E. Knowledge regarding fertility preservation in cancer patients: a population-based survey among Brazilian people during the Pink October awareness event. JBRA Assist Reprod. 2017;21:84-8. PMID: 28609273 DOI: 10.5935/15180557.20170021

Knight S, Lorenzo A, Maloney AM, Srikanthan A, Donen R, Greenblatt E, Gupta A. An approach to fertility preservation in prepubertal and postpubertal females: a critical review of current literature. Pediatr Blood Cancer. 2015;62:935-9. PMID: 25739552 DOI: 10.1002/pbc. 25440

Mahajan N, Patil M, Kaur S, Kaur S, Naidu P. The role of Indian gynecologists in oncofertility care and counseling. J Hum Reprod Sci. 2016;9:179-86. PMID: 27803586 DOI: 10.4103/0974-1208.192061

Mahey R, Kandpal S, Gupta M, Vanamail P, Bhatla N, Malhotra N. Knowledge and awareness about fertility preservation among female patients with cancer: a cross-sectional study. Obstet Gynecol Sci. 2020;63:480-9. PMID: 32689774 DOI: $10.5468 /$ ogs. 20003
Mathur $\mathrm{P}$, Sathishkumar K, Chaturvedi M, Das $\mathrm{P}$, Sudarshan $\mathrm{KL}$, Santhappan S, Nallasamy V, John A, Narasimhan S, Roselind FS; ICMR-NCDIR-NCRP Investigator Group. Cancer Statistics, 2020: Report From National Cancer Registry Programme, India. JCO Glob Oncol. 2020;6:1063-75. PMID: 32673076 DOI: $10.1200 / G 0.20 .00122$

Oktay K, Harvey BE, Partridge AH, Quinn GP, Reinecke J, Taylor HS, Wallace WH, Wang ET, Loren AW. Fertility Preservation in Patients With Cancer: ASCO Clinical Practice Guideline Update. J Clin Oncol. 2018;36:1994-2001. PMID: 29620997 DOI: 10.1200/JCO.2018.78.1914

Parmar V. Rising Incidence of Breast Cancer in the Young Fertile Indian Population-a Reality Check. Indian J Surg Oncol. 2018;9:296-9. PMID: 30287986 DOI: 10.1007/ s13193-018-0800-4

Rashedi AS, de Roo SF, Ataman LM, Edmonds ME, Silva AA, Scarella A, Horbaczewska A, Anazodo A, Arvas A, Ramalho de Carvalho B, Sartorio C, Beerendonk CCM, Diaz-Garcia C, Suh CS, Melo C, Yding Andersen C, Motta E, Greenblatt EM, Van Moer E, Zand E, et al. Survey of Fertility Preservation Options Available to Patients With Cancer Around the Globe. JCO Glob Oncol. 2020;6:331-44. PMID: 32259160 DOI: $10.1200 / J G 0.2016 .008144$

Sallem A, Shore J, Ray-Coquard I, Ferreux L, Bourdon M, Maignien C, Patrat C, Wolf JP, Pocate-Cheriet K. Fertility preservation in women with cancer: a national study about French oncologists awareness, experience, and feelings. J Assist Reprod Genet. 2018;35:1843-50. PMID: 29974370 DOI: $10.1007 / \mathrm{s} 10815-018-1251-0$

WHO - World Health Organization. Cancer. Geneva: WHO; 2020. Available at: https://www.who.int/health-topics/ cancer. Accessed: 7/5/2020.

Woodard TL, Hoffman AS, Covarrubias LA, Holman D, Schover L, Bradford A, Hoffman DB, Mathur A, Thomas J, Volk RJ. The Pathways fertility preservation decision aid website for women with cancer: development and field testing. J Cancer Surviv. 2018;12:101-14. PMID: 29034438 DOI: $10.1007 / \mathrm{s} 11764-017-0649-5$ 